\title{
4 Präzisierung der Fragestellung
}

Vor dem Hintergrund der theoretischen Kontextualisierung sowie dem Desiderat einer adoleszenztheoretischen und biografischen Perspektive mit dem Fokus auf den Körper bei jungen Frauen mit selbstverletzenden Handlungen ergeben sich folgende Fragestellungen, die es im Rahmen dieser Arbeit empirisch zu untersuchen gilt.

Dabei lautet die erkenntnisleitende Hauptfragestellung: „Welche Bedeutung hat der Körper für junge Frauen mit selbstverletzenden Handlungen?“

\section{Operationalisierung der Fragestellung:}

1. Welche psychische und soziale Bedeutung hat der weibliche Körper in Beziehung zur Biografie?

2. Wie werden die puberalen Veränderungen erlebt? Wie wird die adoleszenzspezifische Anforderung, sich mit dem verändernden Körper auseinanderzusetzen, von den jungen Frauen zu bewältigen versucht?

3. Welche Funktionen haben selbstverletzende Handlungen und welche Bedeutung kommt dem Körper dabei zu?

4. Wie beeinflussen kulturelle Geschlechter- und Körperbilder die psychische und soziale Bedeutung des weiblichen Körpers?

In dem anschließenden Kapitel werden das gegenstandsadäquate qualitative Forschungsdesign sowie der Forschungsprozess intersubjektiv nachvollziehbar dargestellt. 